\title{
Can we assign the Borel hulls in a monotone way?
}

by

\author{
Márton Elekes and András Máthé (Budapest)
}

\begin{abstract}
A hull of $A \subseteq[0,1]$ is a set $H$ containing $A$ such that $\lambda^{*}(H)=\lambda^{*}(A)$. We investigate all four versions of the following problem. Does there exist a monotone (with respect to inclusion) map that assigns a Borel $/ G_{\delta}$ hull to every negligible/measurable subset of $[0,1]$ ?

Three versions turn out to be independent of $Z F C$, while in the fourth case we only prove that the nonexistence of a monotone $G_{\delta}$ hull operation for all measurable sets is consistent. It remains open whether existence here is also consistent. We also answer the question of Z. Gyenes and D. Pálvölgyi whether monotone hulls can be defined for every chain of measurable sets. Moreover, we comment on the problem of hulls of all subsets of $[0,1]$.
\end{abstract}

1. Introduction. Let us fix some notation before formulating the problems of this note.

Notation 1.1. Let us denote by $\mathcal{N}, \mathcal{L}, \mathcal{B}$ and $\mathcal{G}_{\delta}$ the classes of Lebesgue negligible, Lebesgue measurable, Borel and $G_{\delta}$ subsets of $[0,1]$, respectively. Let $\lambda$ stand for Lebesgue measure, and $\lambda^{*}$ for Lebesgue outer measure.

Definition 1.2. A set $H \subseteq[0,1]$ is a hull of $A \subseteq[0,1]$ if $A$ is a subset of $H$ and $\lambda^{*}(H)=\lambda^{*}(A)$.

Clearly, every set has a Borel, even a $G_{\delta}$ hull. It is then very natural to ask whether "a bigger set has a bigger hull". (For the two actual motivations of this paper see below.)

Definition 1.3. Let $\mathcal{D}$ and $\mathcal{H}$ be two subclasses of $\mathcal{P}([0,1])$ (usually $\mathcal{D}$ is $\mathcal{N}$ or $\mathcal{L}$, and $\mathcal{H}$ is $\mathcal{B}$ or $\left.\mathcal{G}_{\delta}\right)$. If there exists a map $\varphi: \mathcal{D} \rightarrow \mathcal{H}$ such that

- $\varphi(D)$ is a hull of $D$ for every $D \in \mathcal{D}$,

- $D \subseteq D^{\prime}$ implies $\varphi(D) \subseteq \varphi\left(D^{\prime}\right)$,

then we say that a monotone $\mathcal{H}$ hull operation on $\mathcal{D}$ exists.

2000 Mathematics Subject Classification: Primary 28A51; Secondary 03E15, 03E17, 03E35, 28A05, 28E15, 54H05.

Key words and phrases: hull, envelope, Borel, monotone, Lebesgue, measure, Cohen real, Continuum Hypothesis, add, cof, non, descriptive set theory. 
The four questions we address in this paper are the following.

Question 1.4. Let $\mathcal{D}$ be either $\mathcal{N}$ or $\mathcal{L}$, and let $\mathcal{H}$ be either $\mathcal{B}$ or $\mathcal{G}_{\delta}$. Does there exist a monotone $\mathcal{H}$ hull operation on $\mathcal{D}$ ?

\section{REMARK 1.5.}

- The problem was originally motivated by the following question of Z. Gyenes and D. Pálvölgyi [4]. Suppose that $\mathcal{C} \subseteq \mathcal{L}$ is a chain of sets, i.e. for all $C, C^{\prime} \in \mathcal{C}$ either $C \subseteq C^{\prime}$ or $C^{\prime} \subseteq C$. Does there exist a monotone $\mathcal{B} / G_{\delta}$ hull operation on $\mathcal{C}$ ?

- Another motivation for our set of problems is that it seems to be very closely related to the theory of so called liftings. A map $l: \mathcal{L} \rightarrow \mathcal{L}$ is called a lifting if it preserves $\emptyset$, finite unions and complement, is constant on the equivalence classes modulo nullsets, and maps each equivalence class to one of its members. Note that liftings are clearly monotone. For a survey of this theory see the chapter by Strauss, Macheras and Musiał in [6], or the chapter by Fremlin in [5], or Fremlin [3]. Note that the existence of Borel liftings is known to be independent of $Z F C$, but the existence of a lifting with range in a fixed Borel class is not known to be consistent.

We also remark that liftings are usually considered as $l^{*}: \mathcal{L} / \mathcal{N} \rightarrow \mathcal{L}$ or $l^{*}: \mathcal{P}([0,1]) / \mathcal{N} \rightarrow \mathcal{L}$ maps.

- In light of the theory of liftings it is natural to ask if a monotone Borel $/ G_{\delta}$ hull operation on all of $\mathcal{P}([0,1])$ can be defined. We will see in Section 3 that this is actually equivalent to the existence of a monotone Borel $/ G_{\delta}$ hull operation on $\mathcal{L}$.

REMARK 1.6. We can extend the notion of hull to any uncountable Polish space endowed with a nonzero continuous $\sigma$-finite Borel measure $\mu$. Let $\mu^{*}$ denote the corresponding outer measure. If $\mu$ is finite, then we can define $H$ to be a hull of $A$ if

$$
H \supseteq A \text { and } \mu^{*}(H)=\mu^{*}(A) .
$$

However, if $\mu$ is infinite, then we say that a set $H$ is a hull of $A$ if

$$
H \supseteq A \text { and } \mu^{*}(H \cap I)=\mu^{*}(A \cap I)
$$

for every $\mu$-measurable set $I$. This latter property is in fact equivalent to $\mu(M)=0$ for every $\mu$-measurable set $M \subseteq H \backslash A$.

We remark here that the results (and proofs) of this paper remain valid if we replace $[0,1]$ by $\mathbb{R}$, or by $\mathbb{R}^{n}$, or more generally, by any uncountable Polish space endowed with a nonzero continuous $\sigma$-finite Borel measure. Statement 3.2 is still true in this more general setting, as one can combine Lemma 3.1 with the fact the every such Polish space is Borel isomorphic 
(with a measure preserving isomorphism) either to the real line, or to a subinterval $[a, b]$ of the real line [7].

The paper is organized as follows. First, in the next section we settle the independence of the existence of a monotone Borel $/ G_{\delta}$ hull on $\mathcal{N}$. The consistency of the nonexistence immediately yields the consistency of the nonexistence of a monotone Borel $/ G_{\delta}$ hull on $\mathcal{L}$. Then, in Section 3, we prove that under $C H$ there is a monotone Borel hull on $\mathcal{L}$, and prove partial results concerning $G_{\delta}$ hulls. We conclude the paper by collecting the open questions in Section 4.

2. Monotone hulls for nullsets. Recall that $\operatorname{non}(\mathcal{N})=\min \{|H|$ : $H \subseteq[0,1], H \notin \mathcal{N}\}$, where $|\cdot|$ denotes cardinality. In the following, each cardinal $\kappa$ is identified with its initial ordinal, i.e. with the smallest ordinal of cardinality $\kappa$, and also every ordinal is identified with the set of smaller ordinals. For the standard set theory notation and techniques we use here see e.g. [9] and [1].

TheOREM 2.1. In a model obtained by adding $\omega_{2}$ Cohen reals to a model satisfying $\mathrm{CH}$ there is no monotone Borel hull operation on $\mathcal{N}$.

Proof. We need two well-known facts. Firstly, $\operatorname{non}(\mathcal{N})=\omega_{2}$ in this model [1]. Secondly, in this model there is no strictly increasing sequence of Borel sets of length $\omega_{2}$ (this is proved in [8], see also [2]).

Assume that $\varphi: \mathcal{N} \rightarrow \mathcal{B}$ is a monotone hull operation. Choose $H=\left\{x_{\alpha}\right.$ : $\alpha<\operatorname{non}(\mathcal{N})\} \notin \mathcal{N}$, and consider $\varphi\left(\left\{x_{\beta}: \beta<\alpha\right\}\right)$ for $\alpha<\operatorname{non}(\mathcal{N})$. This is an increasing $\omega_{2}$-long sequence of Borel sets, which cannot stabilize, since then $H$ would be contained in a nullset. But then we can select a strictly increasing subsequence of length $\omega_{2}$, a contradiction.

The following is immediate.

COROLlary 2.2. Under the same assumption there exists no monotone $G_{\delta}$ hull operation on $\mathcal{N}$.

REMARK 2.3. We will see in Remark 3.14 that the length $\omega_{2}$ is optimal in the sense that all shorter well-ordered chains have monotone $G_{\delta}$ hulls.

$\operatorname{Recall}$ that $\operatorname{add}(\mathcal{N})=\min \{|\mathcal{F}|: \mathcal{F} \subseteq \mathcal{N}, \cup \mathcal{F} \notin \mathcal{N}\}$ and $\operatorname{cof}(\mathcal{N})=$ $\min \{|\mathcal{F}|: \mathcal{F} \subseteq \mathcal{N}, \forall N \in \mathcal{N} \exists F \in \mathcal{F}$ such that $N \subseteq F\}$, and also that $\operatorname{add}(\mathcal{N})=\operatorname{cof}(\mathcal{N})$ is consistent [1] (note that e.g. $C H$ implies this equality).

TheOrem 2.4. Assume $\operatorname{add}(\mathcal{N})=\operatorname{cof}(\mathcal{N})$. Then there exists a monotone $G_{\delta}$ hull operation on $\mathcal{N}$.

Proof. Let $\left\{N_{\alpha}: \alpha<\operatorname{cof}(\mathcal{N})\right\}$ be a cofinal family in $\mathcal{N}$, that is, $\forall N \in \mathcal{N}$ $\exists \alpha<\operatorname{cof}(\mathcal{N})$ such that $N \subseteq N_{\alpha}$. For every $\alpha<\operatorname{cof}(\mathcal{N})$ define, using transfinite recursion, $A_{\alpha}=$ a $G_{\delta}$ hull of $\bigcup_{\beta<\alpha} A_{\beta} \cup N_{\alpha}$. Clearly, $\left\{A_{\alpha}: \alpha<\operatorname{cof}(\mathcal{N})\right\}$ 
is a cofinal increasing sequence of $G_{\delta}$ sets. Now, for every $N \in \mathcal{N}$ define $\varphi(N)=A_{\alpha_{N}}$, where $\alpha_{N}$ is the minimal index for which $H \subseteq A_{\alpha_{N}}$. It is easy to see that $\varphi: \mathcal{N} \rightarrow \mathcal{G}_{\delta}$ is a monotone hull operation.

The following is again immediate.

COROLlary 2.5. Under the same assumption there exists a monotone Borel hull operation on $\mathcal{N}$.

3. Monotone hulls for all sets. First we note (Statement 3.2 below) that the title of this section is justified, as there is no difference between working with measurable sets or arbitrary sets.

We need a well-known lemma first. Recall that the density topology of $\mathbb{R}$ consists of those measurable sets that have Lebesgue density 1 at each of their points (see e.g. [10]). Closure in this topology is denoted by $\bar{H}^{d}$, and the term "hull" is used in the sense of Remark 1.6.

LEMma 3.1. $\bar{H}^{d}$ is a hull of $H$ for every $H \subseteq \mathbb{R}$.

Proof. Assume to the contrary that there exists a Lebesgue measurable set $L \subseteq \mathbb{R}$ with $\lambda(L)>0$ such that $L \subseteq \bar{H}^{d} \backslash H$. Set $L_{0}=\{x \in L: x$ is a density point of $L\}$. By the Lebesgue Density Theorem, $L \backslash L_{0}$ is a nullset, which easily implies that $L_{0} \neq \emptyset$ is open in the density topology. But $L_{0} \subseteq \bar{H}^{d}$ is disjoint from $H$, a contradiction.

Statement 3.2. The existence of a monotone Borel $/ G_{\delta}$ hull operation on $\mathcal{P}([0,1])$ is equivalent to the existence of a monotone Borel $/ G_{\delta}$ hull operation on $\mathcal{L}$.

Proof. On the one hand, the restriction to $\mathcal{L}$ of a monotone hull operation on $\mathcal{P}([0,1])$ is itself a monotone hull operation.

On the other hand, by the previous lemma there exists a monotone hull operation $\psi: \mathcal{P}([0,1]) \rightarrow \mathcal{L}$ (note that $[0,1]$ is closed in the density topology). Hence if $\varphi$ is a monotone hull operation on $\mathcal{L}$ then $\varphi \circ \psi$ is a monotone hull operation on $\mathcal{P}([0,1])$.

Theorem 2.1 immediately implies the following.

COROLlary 3.3. In a model obtained by adding $\omega_{2}$ Cohen reals to a model satisfying $C H$ there is no monotone Borel or $G_{\delta}$ hull operation on $\mathcal{L}$.

Now we turn to the positive results.

TheOREm 3.4. Assume CH. Then there is a monotone Borel hull operation on $\mathcal{L}$.

Before we prove this theorem we need a few lemmas. In case $\mathcal{H}=\mathcal{B}$ the first one is a special case of a well-known result about Borel liftings, but there are no such results in the case of $\mathcal{G}_{\delta}$. 
Let us denote by $A \triangle B$ the symmetric difference of $A$ and $B$.

Lemma 3.5. ( $C H)$ There exists a monotone map $\psi: \mathcal{L} \rightarrow \mathcal{G}_{\delta}$ such that $\lambda(M \triangle \psi(M))=0$ for every $M \in \mathcal{L}$, and $\lambda\left(M \triangle M^{\prime}\right)=0$ implies $\psi(M)=$ $\psi\left(M^{\prime}\right)$ for all $M, M^{\prime} \in \mathcal{L}$.

Proof. Let us say that $M, M^{\prime} \in \mathcal{L}$ are equivalent if $\lambda\left(M \triangle M^{\prime}\right)=0$. Denote by $[M]$ the equivalence class of $M$ and by $\mathcal{L} / \mathcal{N}$ the set of classes. We say that $\left[M_{1}\right] \leq\left[M_{2}\right]$ if there are $M_{1}^{\prime} \in\left[M_{1}\right]$ and $M_{2}^{\prime} \in\left[M_{2}\right]$ such that $M_{1}^{\prime} \subseteq M_{2}^{\prime}$.

It is sufficient to define $\Psi: \mathcal{L} / \mathcal{N} \rightarrow \mathcal{G}_{\delta}$ so that $[M] \leq\left[M^{\prime}\right]$ implies $\Psi([M]) \subseteq \Psi\left(\left[M^{\prime}\right]\right)$ for all $M, M^{\prime} \in \mathcal{L}$, and $\Psi([M]) \in[M]$ for every $M \in \mathcal{L}$.

Enumerate $\mathcal{L} / \mathcal{N}$ as $\left\{\left[M_{\alpha}\right]: \alpha<\omega_{1}\right\}$. For every $\alpha<\omega_{1}$ define

$$
\Psi\left(\left[M_{\alpha}\right]\right)=\bigcap_{\substack{\left.\beta<\alpha \\ M_{\beta}\right] \geq\left[M_{\alpha}\right]}} \Psi\left(\left[M_{\beta}\right]\right) \cap\left(\text { a } G_{\delta} \text { hull of } \bigcup_{\substack{\gamma<\alpha \\\left[M_{\gamma}\right] \leq\left[M_{\alpha}\right]}} \Psi\left(\left[M_{\gamma}\right]\right) \cup M_{\alpha}\right) .
$$

It is not hard to check that this is a $G_{\delta}$ set such that $\left[M_{\gamma}\right] \leq\left[M_{\alpha}\right] \leq\left[M_{\beta}\right]$ implies $\Psi\left(\left[M_{\gamma}\right]\right) \subseteq \Psi\left(\left[M_{\alpha}\right]\right) \subseteq \Psi\left(\left[M_{\beta}\right]\right)$, and that $\Psi\left(\left[M_{\alpha}\right]\right) \in\left[M_{\alpha}\right]$, hence the construction works.

REMARK 3.6.

- Actually we will not use the fact that $\psi$ is constant on the equivalence classes.

- We do not know whether $C H$ is needed in this lemma, nor if $C H$ could be replaced by Martin's Axiom.

The following lemma is the only result we can prove for $\mathcal{B}$ but not for $\mathcal{G}_{\delta}$.

Lemma 3.7. (CH) There exists a monotone hull operation $\varphi: \mathcal{N} \rightarrow \mathcal{B}$ such that

(i) $\varphi\left(N \cup N^{\prime}\right) \subseteq \varphi(N) \cup \varphi\left(N^{\prime}\right)$ for all $N, N^{\prime} \in \mathcal{N}$ (subadditivity),

(ii) $\bigcup\{\varphi(N): N \subseteq B, N \in \mathcal{N}\} \backslash B \in \mathcal{N}$ for every $B \in \mathcal{B}$.

Proof. Let $\left\{A_{\alpha}: \alpha<\omega_{1}\right\}$ and $\alpha_{N}$ be as in the proof of Theorem 2.4 $\left(\right.$ note that $\operatorname{add}(\mathcal{N})=\operatorname{cof}(\mathcal{N})=\omega_{1}$ under $\left.C H\right)$. Set $A_{\alpha}^{*}=A_{\alpha} \backslash \bigcup_{\beta<\alpha} A_{\beta}$. Enumerate $\mathcal{B}$ as $\left\{B_{\alpha}: \alpha<\omega_{1}\right\}$ and for every $\alpha<\omega_{1}$ define the countable set

$$
\mathcal{B}_{\alpha}=\left\{\bigcup_{i=0}^{n} B_{\beta_{i}}: n \in \mathbb{N}, \beta_{i}<\alpha(0 \leq i \leq n)\right\} .
$$

Note that every $\mathcal{B}_{\alpha}$ is closed under finite unions.

Now define

$$
\varphi(N)=\bigcup_{\alpha \leq \alpha_{N}}\left(A_{\alpha}^{*} \cap \bigcap_{\substack{B \in \mathcal{B}_{\alpha} \\ N \cap A_{\alpha}^{*} \subseteq B}} B\right)
$$


This is clearly a disjoint union. It is easy to see that $\varphi$ is a monotone Borel hull operation (note that $\varphi(N) \subseteq A_{\alpha_{N}}$ ).

For every $\alpha<\omega_{1}$ define $\varphi_{\alpha}(N)=A_{\alpha}^{*} \cap \varphi(N)(N \in \mathcal{N})$. In order to check subadditivity, let $N, N^{\prime} \in \mathcal{N}$. We may assume $\alpha_{N} \leq \alpha_{N^{\prime}}$, so clearly $\alpha_{N \cup N^{\prime}}=\alpha_{N^{\prime}}$. It suffices to check that each $\varphi_{\alpha}$ is subadditive. If $\alpha>\alpha_{N}$ then actually $\varphi_{\alpha}\left(N \cup N^{\prime}\right)=\varphi_{\alpha}\left(N^{\prime}\right)$, so we are done. Suppose now $\alpha \leq \alpha_{N}$. Let $x \in A_{\alpha}^{*}$ be such that $x \notin \varphi(N) \cup \varphi\left(N^{\prime}\right)$. Then there exist $B \supseteq N \cap A_{\alpha}^{*}$ and $B^{\prime} \supseteq N^{\prime} \cap A_{\alpha}^{*}$ in $\mathcal{B}_{\alpha}$ such that $x \notin B, B^{\prime}$. But then $B \cup B^{\prime} \in \mathcal{B}_{\alpha}$ witnesses that $x \notin \varphi\left(N \cup N^{\prime}\right)$, since $x \notin B \cup B^{\prime} \supseteq\left(N \cup N^{\prime}\right) \cap A_{\alpha}^{*}$.

Finally, to prove (ii) it is sufficient to show that $N \subseteq B_{\alpha}$ implies that $\varphi(N) \backslash B_{\alpha} \subseteq A_{\alpha}$ for every $N \in \mathcal{N}$ and $\alpha<\omega_{1}$. So let $x \in \varphi_{\beta}(N)$ for some $\beta>\alpha$. We have to show $x \in B_{\alpha}$. But this simply follows from the definition of $\varphi$, since $B_{\alpha} \in \mathcal{B}_{\beta}$.

Lemma 3.8. Let $\mathcal{H}$ be either $\mathcal{B}$ or $\mathcal{G}_{\delta}$. Assume that there exists a monotone map $\psi: \mathcal{L} \rightarrow \mathcal{H}$ such that $\lambda(M \triangle \psi(M))=0$ for every $M \in \mathcal{L}$, and also that there exists a monotone hull operation $\varphi: \mathcal{N} \rightarrow \mathcal{H}$ such that

- $\varphi\left(N \cup N^{\prime}\right) \subseteq \varphi(N) \cup \varphi\left(N^{\prime}\right)$ for all $N, N^{\prime} \in \mathcal{N}$,

- $\bigcup\{\varphi(N): N \subseteq H, N \in \mathcal{N}\} \backslash H \in \mathcal{N}$ for every $H \in \mathcal{H}$.

Then $\varphi$ can be extended to a monotone hull operation $\varphi^{*}: \mathcal{L} \rightarrow \mathcal{H}$.

Proof. We may assume that $\psi(N)=\emptyset$ for every $N \in \mathcal{N}$ (by redefining $\psi$ on $\mathcal{N}$ to be constantly $\emptyset$, if necessary).

Define

$$
\varphi^{*}(M)=\psi(M) \cup \varphi(M \backslash \psi(M)) \cup \varphi\left(\bigcup_{\substack{N \subseteq \psi(M) \\ \emptyset \neq N \in \mathcal{N}}} \varphi(N) \backslash \psi(M)\right) .
$$

Clearly $\varphi^{*}(M) \in \mathcal{H}$. As the union of the first two summands contains $M$, we obtain $M \subseteq \varphi^{*}(M)$. Moreover, $\varphi^{*}(M)$ is a hull of $M$, since the first summand is equivalent to $M$ and the last two summands are nullsets. It is also easy to see that $\varphi^{*}$ extends $\varphi$.

We still have to check monotonicity of $\varphi^{*}$. First we prove

$$
N^{\prime} \in \mathcal{N}, M^{\prime} \in \mathcal{L}, N^{\prime} \subseteq \psi\left(M^{\prime}\right) \Rightarrow \varphi\left(N^{\prime}\right) \subseteq \varphi^{*}\left(M^{\prime}\right) .
$$

Indeed, the case $N^{\prime}=\emptyset$ is trivial to check, and otherwise

$$
\begin{aligned}
& \varphi\left(N^{\prime}\right) \subseteq \bigcup_{\substack{N \subseteq \psi\left(M^{\prime}\right) \\
\emptyset \neq N \in \mathcal{N}}} \varphi(N) \subseteq\left(\bigcup_{\substack{N \subseteq \psi\left(M^{\prime}\right) \\
\emptyset \neq N \in \mathcal{N}}} \varphi(N) \backslash \psi\left(M^{\prime}\right)\right) \cup \psi\left(M^{\prime}\right) \\
& \subseteq \varphi\left(\bigcup_{\substack{N \subseteq \psi\left(M^{\prime}\right) \\
\emptyset \neq N \in \mathcal{N}}} \varphi(N) \backslash \psi\left(M^{\prime}\right)\right) \cup \psi\left(M^{\prime}\right) \subseteq \varphi^{*}\left(M^{\prime}\right),
\end{aligned}
$$

which proves (1). 
Let now $M \subseteq M^{\prime}$ be arbitrary elements of $\mathcal{L}$. We need to show that all three summands of $\varphi^{*}(M)$ are contained in $\varphi^{*}\left(M^{\prime}\right)$.

Firstly, $\psi(M) \subseteq \psi\left(M^{\prime}\right)$.

Secondly, define $N^{\prime}=(M \backslash \psi(M)) \cap \psi\left(M^{\prime}\right)$. Using the subadditivity of $\varphi$ and then (1) we obtain

$$
\begin{aligned}
\varphi(M \backslash \psi(M)) & \subseteq \varphi\left((M \backslash \psi(M)) \cap \psi\left(M^{\prime}\right)\right) \cup \varphi\left((M \backslash \psi(M)) \backslash \psi\left(M^{\prime}\right)\right) \\
& \subseteq \varphi\left(N^{\prime}\right) \cup \varphi\left(M^{\prime} \backslash \psi\left(M^{\prime}\right)\right) \subseteq \varphi^{*}\left(M^{\prime}\right) .
\end{aligned}
$$

Thirdly, let

$$
N^{\prime}=\left(\bigcup_{\substack{N \subseteq \psi(M) \\ \emptyset \neq N \in \mathcal{N}}} \varphi(N) \backslash \psi(M)\right) \cap \psi\left(M^{\prime}\right) .
$$

Using the subadditivity of $\varphi$ and then (1) we obtain

$$
\begin{aligned}
& \varphi\left(\bigcup_{\substack{N \subseteq \psi(M) \\
\emptyset \neq N \in \mathcal{N}}} \varphi(N) \backslash \psi(M)\right) \\
& \subseteq \varphi\left(\left(\bigcup_{\substack{N \subseteq \psi(M) \\
\emptyset \neq N \in \mathcal{N}}}^{\subseteq} \varphi(N) \backslash \psi(M)\right) \cap \psi\left(M^{\prime}\right)\right) \cup \varphi\left(\left(\bigcup_{\substack{N \subseteq \psi(M) \\
\emptyset \neq N \in \mathcal{N}}}^{\bigcup} \varphi(N) \backslash \psi(M)\right) \backslash \psi\left(M^{\prime}\right)\right) \\
& \qquad \varphi\left(N^{\prime}\right) \cup \varphi\left(\bigcup_{\substack{N \subseteq \psi\left(M^{\prime}\right) \\
\emptyset \neq N \in \mathcal{N}}}^{\bigcup} \varphi(N) \backslash \psi\left(M^{\prime}\right)\right) \subseteq \varphi^{*}\left(M^{\prime}\right) .
\end{aligned}
$$

This concludes the proof.

Proof of Theorem 3.4. Lemmas 3.5 and 3.7 show that in the case of $\mathcal{H}=\mathcal{B}$ the requirements of Lemma 3.8 can be satisfied, so the proof of Theorem 3.4 is complete.

REMARK 3.9.

- We remark that subadditive monotone maps are actually additive.

- The proof actually gives a monotone $F_{\sigma \delta \sigma}$ hull. However, we do not know whether a monotone $G_{\delta}$ hull operation on $\mathcal{L}$ exists (Question 4.6). Of course, in light of the previous theorem, under $C H$, this is equivalent to assigning $G_{\delta}$ hulls only to the Borel (or $F_{\sigma \delta \sigma}$ ) sets in a monotone way.

QUestion 3.10. Is there a monotone $G_{\delta}$ hull operation on $\mathcal{B}$ ? Or on $\mathcal{F}_{\sigma \delta \sigma}$ ? Or on any other fixed Borel class, e.g. $\mathcal{F}_{\sigma}$ ? (Of course $\mathcal{G}_{\delta}$ and the simpler ones are not interesting.)

Our next goal is to prove Theorem 3.11 below, the partial result we have concerning monotone $G_{\delta}$ hull operations on $\mathcal{L}$. It shows that it is not possible 
to prove in $Z F C$ the nonexistence of $G_{\delta}$ hulls on $\mathcal{L}$ along the lines of Theorem 2.1 , that is, only by considering long chains of sets.

TheOREM 3.11. Assume that there exists a monotone $G_{\delta}$ hull operation $\psi$ on $\mathcal{N}$ (which follows e.g. from $\operatorname{add}(\mathcal{N})=\operatorname{cof}(\mathcal{N}))$. Let $\mathcal{C} \subseteq \mathcal{P}([0,1])$ be a chain of sets, that is, for all $C, C^{\prime} \in \mathcal{C}$ either $C \subseteq C^{\prime}$ or $C^{\prime} \subseteq C$. Then there exists a monotone $G_{\delta}$ hull operation on $\mathcal{C}$.

Proof. By Lemma 3.1 we may assume that $\mathcal{C} \subseteq \mathcal{L}$.

Partition $\mathcal{C}$ into the intervals $\mathcal{I}_{r}=\{C \in \mathcal{C}: \lambda(C)=r\}$. Let $R=\{r \in$ $\left.[0,1]: \mathcal{I}_{r} \neq \emptyset\right\}$, and fix an element $C_{r} \in \mathcal{I}_{r}$ for every $r \in R$. Well-order $R$ as $\left\{r_{\alpha}: \alpha<|R|\right\}$, and set $R_{\alpha}=\left\{r_{\beta}: \beta<\alpha\right\}$.

Now we define $\varphi\left(C_{r_{\alpha}}\right)$ by transfinite recursion as follows. Fix two countable sets $R_{\alpha}^{-} \subseteq\left\{r \in R_{\alpha}: r<r_{\alpha}\right\}$ and $R_{\alpha}^{+} \subseteq\left\{r \in R_{\alpha}: r>r_{\alpha}\right\}$ so that $\forall r \in R_{\alpha}, r<r_{\alpha} \exists r^{\prime} \in R_{\alpha}^{-}$such that $r \leq r^{\prime}<r_{\alpha}$, and similarly, $\forall r \in R_{\alpha}$, $r>r_{\alpha} \exists r^{\prime} \in R_{\alpha}^{+}$such that $r_{\alpha}<r^{\prime} \leq r$. (Note that $R_{\alpha}^{-}$and $R_{\alpha}^{+}$may be singletons or even empty.) Set

$$
\varphi\left(C_{r_{\alpha}}\right)=\left[\mathrm{a} G_{\delta} \text { hull of }\left(C_{r_{\alpha}} \cup \bigcup_{r \in R_{\alpha}^{-}} \varphi\left(C_{r}\right)\right)\right] \cap \bigcap_{r \in R_{\alpha}^{+}} \varphi\left(C_{r}\right) .
$$

It is easy to see that this is a monotone $G_{\delta}$ hull operation on $\left\{C_{r}: r \in R\right\}$.

We may assume that for the hull operation $\psi$ we have $\psi(\emptyset)=\emptyset$. Then we can define a monotone $G_{\delta}$ hull operation $\varphi_{t}$ on $\mathcal{I}_{t}$ for each $t \in R$ as follows. Let

$$
\varphi_{t}(C)=\varphi\left(C_{t}\right) \cup \psi\left(C \backslash C_{t}\right) \quad\left(C \in \mathcal{I}_{t}\right) .
$$

For each $t \in R$ fix a countable set $R_{t}^{++} \subseteq\{r \in R: r>t\}$ so that $\forall r \in R$, $r>t \exists r^{\prime} \in R_{t}^{++}$such that $t<r^{\prime} \leq r$. Set

$$
\varphi(C)=\varphi_{t}(C) \cap \bigcap_{r \in R_{t}^{++}} \varphi\left(C_{r}\right)
$$

for every $C \in \mathcal{I}_{t}$ and every $t \in R$. This is a proper definition since for $C=C_{t}$ this is just an equality. It is easy to check that $\varphi(C)$ is a $G_{\delta}$ hull of $C$ and that $\varphi$ is monotone.

Finally, we prove in $Z F C$ that rather long well-ordered chains have monotone $G_{\delta}$ hulls.

Lemma 3.12. Let $\xi \leq \operatorname{add}(\mathcal{N})$ and $\mathcal{C}=\left\{M_{\alpha}: \alpha<\xi\right\} \subseteq \mathcal{P}([0,1])$ be such that $M_{\alpha} \subseteq M_{\beta}$ for all $\alpha \leq \beta<\xi$. Then there exists a monotone $G_{\delta}$ hull operation on $\mathcal{C}$.

Proof. By Lemma 3.1 we may assume that $\mathcal{C} \subseteq \mathcal{L}$.

By transfinite recursion define $A_{\alpha}$ to be a $G_{\delta}$ hull of the set $M_{\alpha} \cup$ $\bigcup_{\beta<\alpha}\left(A_{\beta} \backslash M_{\alpha}\right)$. Clearly every $A_{\beta} \backslash M_{\alpha}$ is a nullset; moreover, there are $|\alpha|<\operatorname{add}(\mathcal{N})$ many of them; hence $A_{\alpha}$ is a hull of $M_{\alpha}$ too. 
Recall that $\kappa^{+}$is the successor cardinal of $\kappa$ and also that every $\xi<\kappa^{+}$ has a cofinal (i.e. unbounded) subset of order type at most $\kappa$.

Theorem 3.13. Let $\eta<\operatorname{add}(\mathcal{N})^{+}$and $\mathcal{C}=\left\{M_{\alpha}: \alpha<\eta\right\} \subseteq \mathcal{P}([0,1])$ be such that $M_{\alpha} \subseteq M_{\beta}$ for all $\alpha \leq \beta<\eta$. Then there exists a monotone $G_{\delta}$ hull operation on $\mathcal{C}$.

Proof. By Lemma 3.1 we may assume that $\mathcal{C} \subseteq \mathcal{L}$.

We argue by induction on $\eta$. Fix a cofinal subset $X \subseteq \eta$ of order type $\xi \leq \operatorname{add}(\mathcal{N})$ and also a monotone $G_{\delta}$ hull operation $\varphi_{X}:\left\{M_{\alpha}: \alpha \in X\right\} \rightarrow \mathcal{G}_{\delta}$ by the previous lemma. Every complementary interval $[\beta, \gamma)$ of $X$ (i.e. every interval that is maximal disjoint from $X$ ) is of order type $<\eta$, hence by the inductive assumption there exists a monotone $G_{\delta}$ hull operation $\varphi_{[\beta, \gamma)}:\left\{M_{\alpha}: \alpha \in[\beta, \gamma)\right\} \rightarrow \mathcal{G}_{\delta}$. Also fix a measure zero $G_{\delta}$ hull $H_{[\beta, \gamma)}$ of $\bigcup_{\delta<\beta, \delta \in X}\left(\varphi_{X}\left(M_{\delta}\right) \backslash M_{\beta}\right)$. Now for every $[\beta, \gamma)$ and every $\alpha \in[\beta, \gamma)$ define

$$
\varphi\left(M_{\alpha}\right)=\left(H_{[\beta, \gamma)} \cup \varphi_{[\beta, \gamma)}\left(M_{\alpha}\right)\right) \cap \varphi_{X}\left(M_{\gamma}\right),
$$

and also define $\varphi\left(M_{\alpha}\right)=\varphi_{X}\left(M_{\alpha}\right)$ for every $\alpha \in X$. It is then easy to see that this is a monotone $G_{\delta}$ hull operation on $\mathcal{C}$.

REMARK 3.14. As $\operatorname{add}(\mathcal{N}) \geq \omega_{1}$, we see that the length $\omega_{2}$ of the chain in the proof of Theorem 2.1 was optimal.

4. Concluding remarks and open problems. First we show (in $Z F C$ ) that there are no strictly monotone hulls of any kind.

Statement 4.1. There is no $\subsetneq$-preserving monotone Borel hull on $\mathcal{N}$.

Proof. It is well known that in every infinite set of size $\kappa$ there is a chain (of subsets) of size greater than $\kappa$. Indeed, let $\lambda=\min \left\{\lambda^{\prime}: 2^{\lambda^{\prime}}>\kappa\right\}$, and let us consider $X=\left\{x \in 2^{\lambda}: \exists \alpha<\lambda \forall \beta \in[\alpha, \lambda) x(\beta)=0\right\}\left(2^{\lambda}\right.$ is considered as the set of functions from $\lambda$ to $2=\{0,1\})$. Then $|X|=\kappa$, and it suffices to produce a $2^{\lambda}$-sized chain of subsets of $X$. Let $<_{\text {lex }}$ denote the lexicographic ordering on $2^{\lambda}$, and for $y \in 2^{\lambda}$ set $A_{y}=\left\{x \in X: x \leq_{\operatorname{lex}} y\right\}$. Then $y<_{\operatorname{lex}} y^{\prime}$ implies $A_{y} \subsetneq A_{y^{\prime}}$, so $\left\{A_{y}: y \in 2^{\lambda}\right\}$ is a chain of size $2^{\lambda}>\kappa$.

Hence the usual middle-third Cantor set (which is of measure zero) contains a chain of size greater than continuum; but then the Borel hulls of the elements of this chain form more than continuum many Borel sets, which is impossible.

Now we pose a few somewhat vague problems, some of which may turn out to be easy. 
Question 4.2. It would be interesting to know what happens

- if we look at the category analogue of Question 1.4, that is, when $\mathcal{N}$ and $\mathcal{L}$ are replaced by the first Baire category (= meager) sets and the sets with the property of Baire;

- if we require that our monotone hulls are translation or isometry invariant.

Question 4.3. Does Theorem 3.4 remain valid if we replace CH by Martin's Axiom? That is, does there exist a monotone Borel hull operation on $\mathcal{L}$ if we assume Martin's Axiom?

We now repeat the open questions of the paper for the sake of completeness.

QUESTION 4.4. Is there $\left(\right.$ in ZFC) a monotone map $\psi: \mathcal{L} \rightarrow \mathcal{G}_{\delta}$ such that $\lambda(M \triangle \psi(M))=0$ for every $M \in \mathcal{L}$ ? If yes, is there one such that $\lambda\left(M \triangle M^{\prime}\right)=0$ implies $\psi(M)=\psi\left(M^{\prime}\right)$ for all $M, M^{\prime} \in \mathcal{L}$ ?

QUESTION 4.5. Is there a monotone $G_{\delta}$ hull operation on $\mathcal{B}$ ? Or on $\mathcal{F}_{\sigma \delta \sigma}$ ? Or on any other fixed Borel class, e.g. $\mathcal{F}_{\sigma}$ ? (Of course $\mathcal{G}_{\delta}$ and the simpler ones are not interesting.)

Let us conclude with the most important open question.

QUESTION 4.6. Is it possible to assign $G_{\delta}$ hulls to all (measurable) subsets of $[0,1]$ in a monotone way?

Acknowledgements. The authors are indebted to Miklós Laczkovich and Alain Louveau for some helpful comments. We also gratefully acknowledge the support of Öveges Project of ${ }^{N}$ NKTH and KPI.

Research of M. Elekes was partially supported by Hungarian Scientific Foundation grants no. 49786, 61600 and F 43620.

Research of A. Máthé was partially supported by Hungarian Scientific Foundation grants no. T 49786 and T 72655.

\section{References}

[1] T. Bartoszyński and H. Judah, Set Theory: On the Structure of the Real Line, A. K. Peters, Wellesley, MA, 1995.

[2] M. Elekes and K. Kunen, Transfinite sequences of continuous and Baire class 1 functions, Proc. Amer. Math. Soc. 131 (2003), 2453-2457.

[3] D. H. Fremlin, Measure Theory, Vol. 3, Torres Fremlin, 2004.

[4] Z. Gyenes and D. Pálvölgyi, private communication, 2004.

[5] Handbook of Boolean Algebras, Vol. 3, J. D. Monk and R. Bonnet (eds.), NorthHolland, 1989.

[6] Handbook of Measure Theory, E. Pap (ed.), North-Holland, 2002.

[7] A. S. Kechris, Classical Descriptive Set Theory, Springer, 1995. 
[8] K. Kunen, Inaccessibility properties of cardinals, Doctoral Dissertation, Stanford, 1968.

[9] —, Set Theory. An Introduction to Independence Proofs, Stud. Logic Found. Math. 102, North-Holland, 1980.

[10] J. C. Oxtoby, Measure and Category. A Survey of the Analogies between Topological and Measure Spaces, 2nd ed., Grad. Texts in Math. 2, Springer, 1980.

M. Elekes

Rényi Alfréd Institute of Mathematics

Hungarian Academy of Sciences

P.O. Box 127

H-1364 Budapest, Hungary

E-mail: emarci@renyi.hu

http://www.renyi.hu/ emarci
A. Máthé

Eötvös Loránd University Department of Analysis Pázmány Péter sétány 1/c H-1117 Budapest, Hungary E-mail: amathe@cs.elte.hu http://amathe.web.elte.hu

Received 2 July 200\%;

in revised form 12 June 2009 